\section{Origin of the Third Heart Sound}

For many years there has been speculation about the origin of the third heart sound. Some authors have held the theory that it is the sound of ventricular filling. ${ }^{1-3}$ Others have considered the sound to originate from the mitral valve, whether representing early closure of the valve ${ }^{4}$ or due to sudden tautening of the valve and its chordal attachments in early diastole. ${ }^{6}{ }^{7}$

Recently J. S. Fleming, ${ }^{8}$ in a study of mitral regurgitation before and after mitral valve replacement, produced evidence suggesting that the third heart sound in this condition may be due to sudden tautening of the valve in early diastole. Ultrasound measurements indicated that at the time of the sound the valve was in the half-open position, while the apex cardiogram showed abrupt checking of outward movement of the apex of the heart. He suggested that the latter is due to a suddent limitation of downward movement imposed by the tautened mitral valve with its chordal attachments. After excision of the mitral valve and replacement by a StarrEdwards prosthesis the third sound disappeared, and apex cardiography no longer showed a checking movement in early diastole. This might support the theory that intact chordae tendineae are necessary for the production of the third heart sound. Disappearance of the sound might alternatively have resulted from the relief of mitral incompetence, with abolition both of the abnormally large left atrioventricular gradient in early diastole and of the increased stroke output of the left ventricle.

Different combinations of factors in different conditions may contribute to the variable nature of the third heart sound. When the mitral valve is diseased from rheumatism and the chordae tendineae thickened and shortened, it seems possible that sudden tensing of the valve apparatus may be an important factor in the production of the third sound, which in this condition may be exceptionally loud and sharp in character. In severe aortic incompetence closure of the mitral valve in early-to-mid-diastole has been shown to occur, ${ }^{9-11}$ and this may in part be responsible for a loud third sound that may persist for many years. In constrictive pericarditis, on the other hand, where a special knocking form of third heart sound occurs, full dilatation of the ventricle is prevented. It is unlikely, therefore, that the third heart sound in this condition is produced by tautening of the chordae. It is more probable that it is a water-hammer effect resulting from sudden arrest, by the rigid pericardial casing, of rapid early ventricular filling consequent on the steep atrioventricular gradient. ${ }^{12}$ The dull, low-pitched third heart sound heard in hypertensive heart failure may well be no more than the reflection of a raised left atrioventricular pressure gradient in

1 Potain, P. C. E., Semaine Médicale, 1900, 20, 175.

- Crevasse, L., Wheat, M. W., Wilson, J. R., Leeds, R. F., and Taylor, W. J., Circulation, 1962, 25, 635 .

- Kuo, P. T., Hildreth, E. A., and Kay, C. F., Annals of Internal Medicine, $1951,35,1306$.

- Gibson, A. G., Lancet, 1907, 2, 1380.

- Thayer, W. S., Archives of Internal Medicine, 1909, 4, 297

- Dock, W., Circulation, 1959, 19, 376.

' Nixon, P. G. F., British Heart fournal, 1961, 23, 677.

- Fleming, J. S., British Heart fournal, 1969, 31, 192.

- Wright, J. L., Toscano-Barboza, E., and Brandenburg, R. O., Proceedings of the Staff Meetings of the Mayo Clinic, 1956, 31, 120.

Welch, G. H., jun., Braunwald, E., and Sarnoff, S. J., Circulation Research, 1957, 5, 546.

1 Dodge, H. T., Sandler, H., and Evans, T., Circulation, 1960, 22, 741.

12 Mounsey, P., British Heart fournal, 1955, 17, 143. 1 Mourdiinis, Ath., Olsen, E., Raphael, M. J., and Mounsey, J. P. D. early diastole-in fact, a mild water-hammer phenomenon similar to that in constrictive pericarditis.

Still another type of third heart sound is that heard in ventricular aneurysm, which may persist for many years and does not necessarily carry a poor prognosis. Possibly an important factor here may be the thinned portion of ventricular wall transmitting vibrations within the chamber more freely to the exterior. ${ }^{13}$ Finally, the third heart sound in healthy persons under 40 and its disappearance after this age are phenomena whose mechanism is not fully understood. There is evidence that myocardial relaxation is an active process, the ventricle sucking in blood in early diastole. It is possible that the sequential interaction of the forces of active and passive ventricular filing is another factor that may contribute to the production of the third heart sound in health and also in disease.

\section{Cancer and the Nervous System}

A glaring gap in our knowledge is the mechanism of generalized wasting associated with malignant neoplasia. A lead may come from studying the distant effects of carcinoma on the neuromuscular system, pioneered by the late Lord Brain and colleagues, who named this group of conditions the carcinomatous neuromyopathies. ${ }^{1}$ It is fitting that this research continues at the London Hospital, ${ }^{2}$ where many of them were delineated.

A carcinoma or reticulosis may, without secondary deposits, profoundly affect neurones, myelin, muscle, or the myoneural junction. The diversity of clinical syndromes include a myopathy, a myasthenic syndrome, a sensory or mixed motor and sensory neuropathy, anterior-horn cell disease mimicking motor neurone disease, subacute cerebellar atrophy, and progressive leucoencephalopathy. The central or peripheral nervous system may be affected at various levels, singly or in combination. These neurological syndromes are not rare. Brain and R. A. Henson ${ }^{1}$ estimated an incidence of $6 \%$ in all patients with a carcinoma. About half the affected patients have neurological symptoms or signs before the diagnosis of neoplasm has been made. Unfortunately removal of the growth, even completely, often fails to reverse the neurological damage. ${ }^{2}$

Carcinomatous myopathy affects the muscles of the limb girdles and trunk. Patients have difficulty in turning in bed, rising from the lying or sitting position, and negotiating stairs, because trunk and pelvic-girdle muscles are weak. Combing hair or hanging washing on a line may become impossible for a woman or shaving for a man because of weakness in the shoulder muscles. Muscular fatigue at times responds, partially or completely, to treatment with an anticholinesterase and hence resembles myasthenia gravis. Clinically there is a close resemblance to thyrotoxic myopathy, myasthenia gravis, dermatomyositis, or polymyositis. The diagnosis of carcinomatous myopathy should be considered when the patient also has dysaesthesiae or the tendon reflexes are depressed beyond the field of weakness. ${ }^{1}$

A pure sensory neuropathy was first described by $D$. Denny-Brown. ${ }^{3}$ Neurones of the posterior root ganglia are primarily affected, with consequent degeneration of the peri- 
pheral sensory nerves and the posterior columns of the spinal cord. Patients complain of painful dysaesthesiae and gradually develop sensory loss that results in ataxia and often complete disablement. The sensory disturbance may involve the face and trunk, and all tendon reflexes disappear. The high incidence of bronchial carcinoma, even in women, is stressed in a recent review. ${ }^{2}$ An exactly similar clinical and pathological syndrome can occur in diabetes mellitus. ${ }^{4}$ There was no remission in any case, though arrest was noted in one patient with a neoplasm.

More frequent than a pure sensory neuropathy is a mixed motor and sensory neuropathy, particularly as a complication of a bronchial carcinoma. As in other carcinomatous neuropathies the clinical picture is often not clear-cut, and dementia or extensor plantar responses may be present. Of 19 patients, ${ }^{2}$ nine showed steady deterioration, five remained stationary, and two improved to a limited extent. Death in the majority was due to the direct effects of the neoplasm but one patient died from bulbar palsy. Diagnosis can be difficult because the causes of peripheral neuropathy are legion and the neurological illness may precede the discovery of the neoplasm by many months -2 to 59 months in $\mathbf{P}$. B. Croft and $M$. Wilkinson's series. ${ }^{2}$

A neurological disorder simulating motor neurone disease occurs with bronchial and gastric neoplasia in men and with malignant tumours of the breast in women. The patients showed fasciculation, weakness, and wasting of the bulbar and limb muscles, but the tendon reflexes were not invariably increased as in classical motor neurone disease. Deterioration was slower than in the classical disease but patients died rapidly from the direct effects of the tumour. However, one out of 11 patients improved after removal of the carcinoma. ${ }^{5}$

Subacute cerebellar degeneration occurs most frequently in women with carcinoma of ovary or breast and with bronchial neoplasm in men. The association between ovarian carcinoma and this type of cerebellar degeneration is particularly high. ${ }^{\circ}$ Purkinje cells were diminished in number or completely lost and the molecular layer of the cerebellum narrowed. In addition the direct cerebellar tract was degenerate. Clinically the limbs, trunk, and speech were ataxic, but nystagmus was not prominent. These neurological symptoms preceded the diagnosis of the neoplasm in about half the patients by a period ranging from two months to two years. In the remainder the cerebellar deficiency became manifest when, or after, the diagnosis of the neoplasm had been made. Removal of the tumour failed to influence the cerebellar syndrome.

A rare complication of Hodgkin's disease and chronic lymphatic leukaemia is a progressive disorder affecting the white matter of the cerebral hemispheres aptly called progressive multifocal leucoencephalopathy. ${ }^{7}$ Extensive demyelination affects particularly the occipital lobes, and bizarre

Brain, Sir R., and Henson, R. A., Lancet, 1958, 2, 971.

2 Croft, P. B., and Wilkinson, M., Brain, 1969, 92, 1.

3 Denny-Brown, D., fournal of Neurology, Neurosurgery, and Psychiatry, 1948, 11, 73 .

- Bosanquet, F. D., and Henson, R. A., Folia Psychiatrica, Neurologica, et Neurochirurgica Neerlandica, 1957, 60, 107.

5 Brain, Lord, Croft, P. B., and Wilkinson, M., Brain, 1965, 88, 479.

6 Brain, Lord, and Wilkinson, M., Brain, 1965, 88, 465.

7 Aström, K. B., Mancall, E. L., and Richardson, E. P., Brain, 1958, 81, 93.

${ }^{8}$ Hutchinson, E. C., Leonard, B. J., Maudsley, C., and Yates, P. O., Brain, 1958, 81, 75 .

- Mancall, E. L., and Rosales, R. K., Brain, 1964, 87, 639.

10 Waldenström, J. G., Monoclonal and Polyclonal Hypergammaglobulinemia : Clinical and Biological Significance, 1968, pp. 156-162. Cambridge University Press. gigantic astrocytes are found. Symptoms appear as early as eight months or as late as 15 years after the original diagnosis has been made. The patients present with defects of the visual field, language disorders, pyramidal signs, and dementia. Though patients may fluctuate in levels of consciousness, deterioration is progressive and inexorable. Spinal cord changes have also been described in Hodgkin's disease, other reticuloses, ${ }^{8}$ and carcinoma. ${ }^{9}$

Mental changes may be found in patients with any type of carcinomatous neuropathy. Dementia is commonly associated with the cerebellar syndrome but also occurs with peripheral neuropathy. Agitation and depression have been noted, but whether the incidence is greater than in other forms of organic neurological disease has not been ascertained. ${ }^{1}$

These distant effects of a neoplasm on the nervous system are reminiscent of the hormonal disturbances associated with a variety of growths. ${ }^{10}$ The stage is now set when laboratory research may prove to be fruitful. On the other hand, as so often in research, an entirely different problem may be illuminated. For example, we know that neurones of the cerebellum are selectively vulnerable to alcohol, anoxia, heat stroke, thyroid deficiency, organic mercurials, and, from the studies referred to here, carcinoma of the lung and ovary. Elucidation of this neuronal vulnerability may indicate the cause of more common diseases such as the presenile dementias and motor neurone disease. Nevertheless these studies show that clinical observation and research can still add lustre to modern medicine.

\section{"Medical Register's" Face-lift}

One of the accepted antidotes to middle-age spread and the low spirits it induces is a visit to the beauty parlour, from which a woman at least may emerge feeling rejuvenated. With this sound principle to guide it the General Medical Council has redesigned the Medical Register so that despite its expanding girth and more complicated text it comes out this year with a much improved lay-out. The new edition contains the names of 114,278 medical men and women, of whom some 53,000 were registered in London for England and Wales, 22,000 in Scotland, 13,000 in Ireland, 24,000 in the Commonwealth, and 2,000 in foreign countries. The complexities of registration as well as the numbers of doctors registered have increased considerably since the first Medical Register was issued in 1859 in a single volume. In 1949 the Register went into two volumes for the first time, and in 1968 it began its present occuipation of three. Today we have provisionally and fully registered practitioners, and Commonwealth and foreign practitioners; there are also temporarily registered practitioners, whose names are not included in the lists.

Instead of separate sections in the Register for the various types of registration the G.M.C. now provides a continuous alphabetical list. Against each practitioner's name is a letter designating the place where he was initially registered, while an asterisk distinguishes those who are only provisionally registered. Details of address and registrable qualifications are given as usual. The result is a work of reference much easier to use and for the presbyopic clearer to read. 